\title{
Soft X-Ray Laser Gain from Neon like Gallium and Germanium
}

\author{
Mohammad Z. Mansour ${ }^{1}$, Wessameldin S. Abdelaziz' ${ }^{1}$, Tharwat M. El Sherbini2 \\ ${ }^{1}$ National Institute of Laser Enhanced Sciences, Cairo University, Giza, Egypt \\ ${ }^{2}$ Laboratory of Lasers and New Materials, Faculty of Science, Cairo University, Giza, Egypt \\ Email:wessamlaser@yahoo.com
}

Received 25 April 2016; accepted 23 May 2016; published 26 May 2016

Copyright (C) 2016 by authors and Scientific Research Publishing Inc.

This work is licensed under the Creative Commons Attribution International License (CC BY).

http://creativecommons.org/licenses/by/4.0/

(c) (i) Open Access

\begin{abstract}
Gain coefficients are calculated for neon-like gallium and germanium ions. Shorter wavelengths are calculated and predicted to be emitted. The gain coefficients are calculated among 457 energy levels of the neon-like ions. Collisional excitations were calculated through the distorted wave approximations through five electron temperatures $T_{e}=300,500,700,1000$ and $1500 \mathrm{eV}$.
\end{abstract}

\section{Keywords}

Gain Coefficients, X-Ray Lasers, Collisional Radiative Model

\section{Introduction}

It was suggested that coherent X-ray emission could be produced in the collision of highly energetic electrons with multiply charged ions [1]. The first soft X-ray laser amplification was demonstrated in neon-like sellenium [2] in which a high energy neodymium glass laser is used to strike a thin foil of selenium where it was evaporize and create plasma of neon-like selenium. The high energy electrons in the plasma collide and excite the neon-like selenium ions in the plasma and emit a coherent soft X-ray transition with wavelength $21 \mathrm{~nm}$ [3]. Shortly afterwords a lot of experimentally work was done to check the possibility of the emission of laser radiation from different ions. The authors have done a lot of work to calculate the atomic properties and predict the possible laser transitions from different ions [4]-[6]. Recently a laser transitions from Ne-like Ti, V, Cr, Fe and Co were observed [7].

In this paper, we apply the electron excitation model to predict new gain lines from neon like gallium and germanium, comparing the results with the experimental work.

\section{Calculation of Level Population}

The gain coefficient $g(v)$ of a medium is related to the intensity of the radiation as follow 


$$
I=I_{o} \mathrm{e}^{g(v) z}
$$

where $I_{o}$ is the initial incident intensity of the radiation and $I$ is the intensity after length $z$. In high density plasma, Doppler broadening is the dominant type of broadening [8]-[10] and the gain coefficient $g(v)$ can be given by [11]

$$
g^{D}(v)=\frac{\lambda^{3}}{8 \pi \eta^{3}} g_{j} A_{j i} \sqrt{\frac{M_{i}}{2 \pi k T_{i}}}\left(\frac{N_{j}}{g_{j}}-\frac{N_{i}}{g_{i}}\right)
$$

where we have used $\lambda=\frac{c}{v \eta}$ and $\lambda$ is the transition wavelength, $\eta$ is the refractive index $\eta=\sqrt{1-\frac{n_{e}}{n_{c}}}$ where $n_{c}=\frac{\epsilon_{0} m_{e}}{e^{2}}(2 \pi v)^{2}, g_{i}$ and $g_{j}$ are the statistical weights of the lower and upper levels respectively, $A_{j i}$ is the transition rate, $M_{i}$ is the ion mass, $T_{i}$ is the ion temperature and $N_{i}$ and $N_{j}$ are the lower and upper level populations.

Taking the electron impact excitation and deexcitation as the main mechanism for the population of states we could define our rate equation to be

$$
\frac{\mathrm{d} n_{i}}{\mathrm{~d} t}=-n_{i} \sum_{\substack{j \neq i \\ j>i}}^{l} n_{e} C_{i j}^{e}+\sum_{\substack{j \neq i \\ j>i}}^{l} n_{j}\left(A_{j i}+n_{e} C_{j i}^{d}\right)
$$

where $C_{i j}^{e}$ and $C_{j i}^{d}$ are the excitation and deexcitation rates respectively. Solving Equation (3) for the quasi steady states $\frac{\mathrm{d} n_{i}}{\mathrm{~d} t}=0$, and finding the fractional population $n_{i}$.

In order to determine the population of states we need to determine the total number of atoms $N_{a}$ and to do that we use the same properties of the first experiment of the neon like Selenium. In that experiment a collimated beam of high power laser was cocenterated on a cylinderical target with length $1.2 \mathrm{~cm}$ and $200 \mu \mathrm{m}$ in diameters [12] [13] using the ratio of the ion temperature $T_{i}$ to the electron temperature $T_{e}$ to be 0.4 as in [14] and in a good agreement with [8]. Five electron temperatures were taken for each ion.

The total number of atoms $N_{a}$ could be determined as

$$
N_{a}=\frac{\rho V}{A} \text {, and } N_{I}=f N_{a}
$$

where $\rho$ is the target density, $V$ is the volume, $A$ is the atomic weight of the atoms, $N_{I}$ is the population of ions in the ionization stage $I$ and $f$ is the ion fraction. The fraction of the neon like atoms that was produced in the plasma was taken to be $3 \times 10^{-6}$. Our intiuation behind that ratio was the He-Ne laser, the laser emission from Helium-Neon laser was due to the Neon atoms while their population was very low comparing with the Helium gas as $N_{\mathrm{Ne}} \ll N_{\mathrm{He}}$.

All our calculations were done by our code written in python programming language where we put Equation (3) in the matrix form and apply the boundary condition Equation (4) to solve the equations for $n_{i} / N_{a}$. We used the flexible atomic code (FAC) [15] which is fully relativistic in calculating the energy levels and the effective collision strength using the distorted wave approximation.

Practically the cross section averaged over the velocity distribution of the scattered electrons is very important and is called the rate coefficient and has the form

$$
C_{j i}^{d}=\langle\sigma v\rangle=\int_{0}^{\infty} \sigma_{j i}(E) v(E) f(E) \mathrm{d} E,
$$

as a function of energy. The cross section and the velocity of the scattered electrons could be expressed as

$$
v(E)=\sqrt{2 E / m}, \quad \sigma_{j i} \frac{h^{2}}{8 \pi m E} \frac{\Omega_{j i}}{g_{j}},
$$

and the Maxwellian distribution function is given by 


$$
f(E)=2 \sqrt{\frac{E}{\pi}} \frac{1}{\left(k T_{e}\right)^{3 / 2}} \mathrm{e}^{-E / k T_{e}} .
$$

Substituting in Equation (5) we get

$$
C_{j i}^{d}=\frac{h^{2}}{8 \pi m g_{j}} \sqrt{\frac{2}{m}} \frac{2}{\sqrt{\pi}\left(k T_{e}\right)^{3 / 2}} \int_{0}^{\infty} \Omega_{j i}(E) \mathrm{e}^{-E / k T_{e}} \mathrm{~d} E,
$$

where the integral usually defined as the effective collision strength $\gamma_{j i}$ is

$$
\gamma_{j i}=\int_{0}^{\infty} \Omega_{j i}(E) \mathrm{e}^{-E / k T_{e}} \mathrm{~d}\left(\frac{E}{k T_{e}}\right),
$$

where it is the Maxwellian average of the collision strength. Equation (8) becomes

$$
\begin{aligned}
C_{j i}^{d} & =\frac{2 h^{2}}{8 \pi m} \sqrt{\frac{2}{\pi m}} \frac{1}{g_{j} \sqrt{T_{e}}} \gamma_{j i} \\
& =\frac{8.6287 \times 10^{-6}}{g_{j} \sqrt{T_{e}}} \gamma_{j i} \quad \text { in cgs units }\left(\mathrm{cm}^{3} / \mathrm{s}\right),
\end{aligned}
$$

where $C_{j i}^{d}$ is the collisional de-excitation rate expressed as a function of the effective collision strength $\gamma_{j i}$, the statistical weight $g_{j}$ of the upper state and the electron temperature $T_{e}$. By the same way the excitation rate coefficient $C_{i j}^{e}$ could be expressed as [16]

$$
C_{i j}^{e}=\frac{8.6287 \times 10^{-6}}{g_{i} \sqrt{T_{e}}} \mathrm{e}^{-E_{j i} / k T_{e}} \gamma_{j i} \text { in cgs units }\left(\mathrm{cm}^{3} / \mathrm{s}\right),
$$

where $E_{j i}$ is the energy difference between the initial and final states of the transitions. In the derivation we have used ( $\Omega_{i j} \approx \Omega_{j i}$ ) and subsequently $\left(\gamma_{i j} \approx \gamma_{j i}\right.$ ). Both excitation and de-excitation rates could be expressed as

$$
C_{j i}^{d}=C_{i j}^{e} \frac{g_{i}}{g_{j}} \mathrm{e}^{E_{j i} / k T_{e}} .
$$

Gain coefficient $g(v)$ is related to the cross section $\sigma$ as follow

$$
g(v)=N_{j} \sigma_{s}-N_{i} \sigma_{a}=N_{j} \sigma_{s} \mathcal{F},
$$

where $\mathcal{F}$ is the population inversion factor and can be expressed as

$$
\mathcal{F}=1-\frac{N_{i}}{N_{j}} \frac{\sigma_{a}}{\sigma_{s}}=1-\frac{N_{i}}{N_{j}} \frac{g_{j}}{g_{i}},
$$

where $\sigma_{s}$ and $\sigma_{a}$ are the stimulated and the absorption cross sections, $N_{i}$ and $N_{j}$ are the population densities of states $|i\rangle$ and $|j\rangle$ respectively and $g_{i}$ and $g_{j}$ are the statistical weights of states $|i\rangle$ and $|j\rangle$ respectively.

During the laser pumping, the population inversion should occurs and then the population of the higher energy levels becomes greater than the lower ones $\left(N_{j} N_{i}\right)$ and subsequently the inversion factor decrease and becomes less than unity as shown from Equation (14).

\section{Results and Discussion}

The atomic data of the gallium and germanium ions were relativistically calculated using FAC v1.1.3 [15] and the effective collision strengths were determined using the distorted wave approximations. Five electron temperatures $\left(T_{e}=300,500,700,1000\right.$ and $\left.1500 \mathrm{eV}\right)$ were taken for each ion and the gain coefficients were calculated. A wide range of the electron densities were taken $\left(n_{e}=1 \times 10^{16}\right.$ to $\left.9 \times 10^{23}\right)$.

The resulted curves have the same behavior for the different electron temperatures, so the population inversion $\mathcal{F}$, effective collision strength $\gamma_{j i}$ and gain $g(v)$ at electron temperature $T_{e}=700 \mathrm{eV}$ for both ions are shown in Figure 1 and Figure 2. 


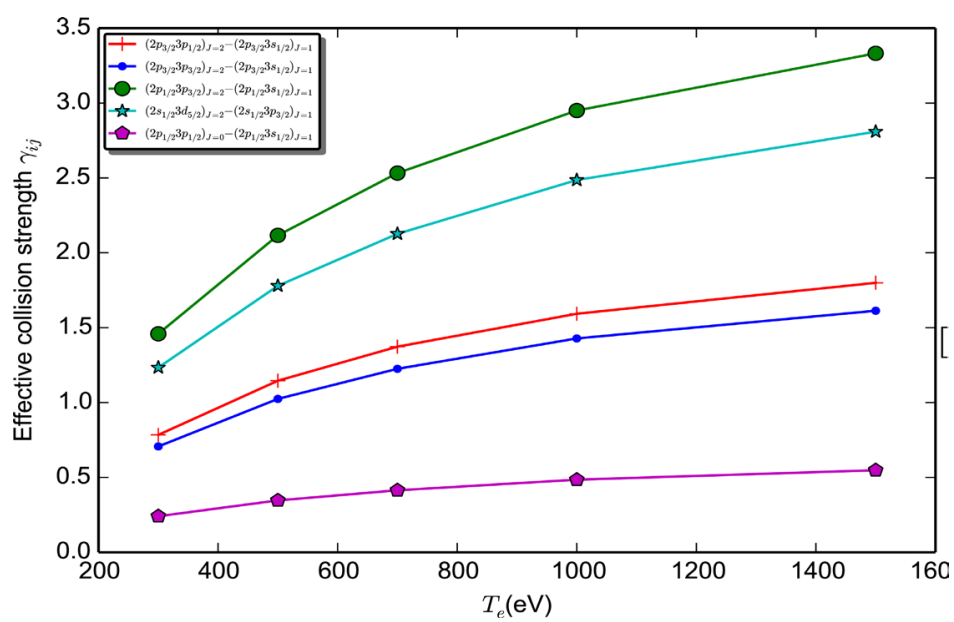

(a)

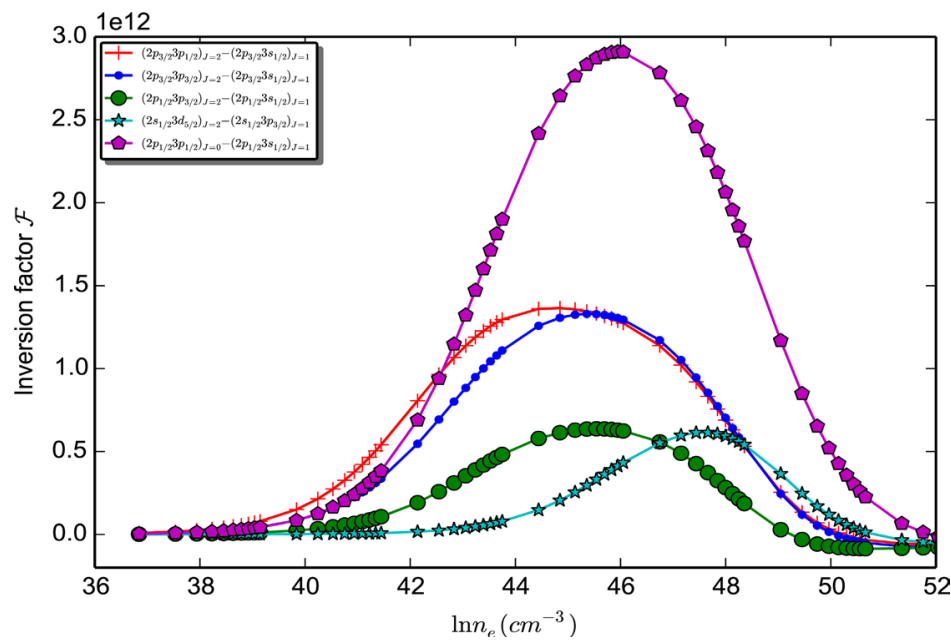

(b)

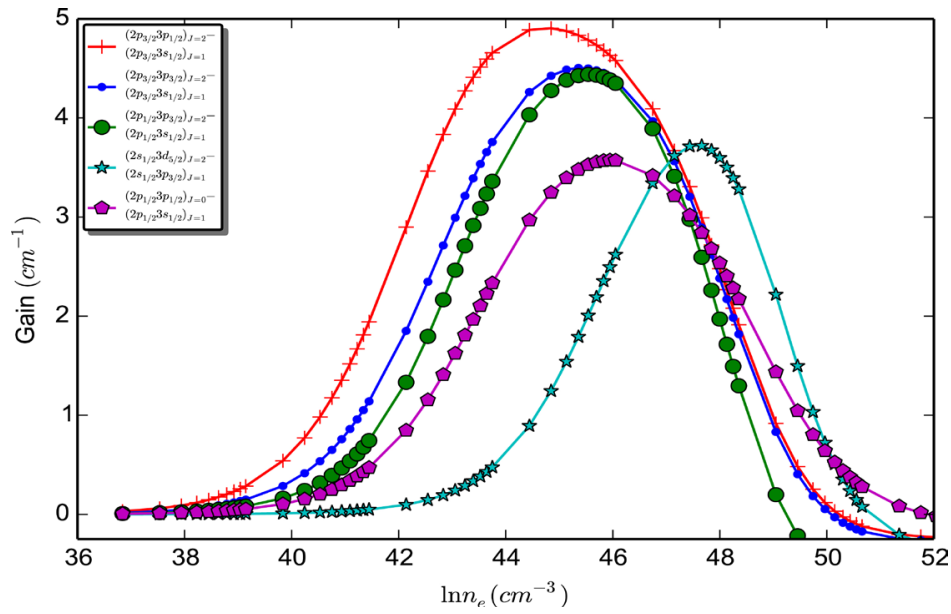

(c)

Figure 1. Effective collision strength, population inversion and gain coefficients for neon-like gallium ions $\mathrm{Ga}^{+21}$ at electron temperatures $T_{e}=700 \mathrm{eV}$. (a) Effective collision strengths of the Ne-like Ga at electron plasma temperatures $T_{e}=700 \mathrm{eV}$; (b) Population inversion factor $\mathcal{F}$ versus electron density $n_{e}$ at electron temperature $T_{e}=700 \mathrm{eV}$; (c) Gain coefficients at $T_{e}=700 \mathrm{eV}$ of the highest gain transitions in Neon like Gallium. 


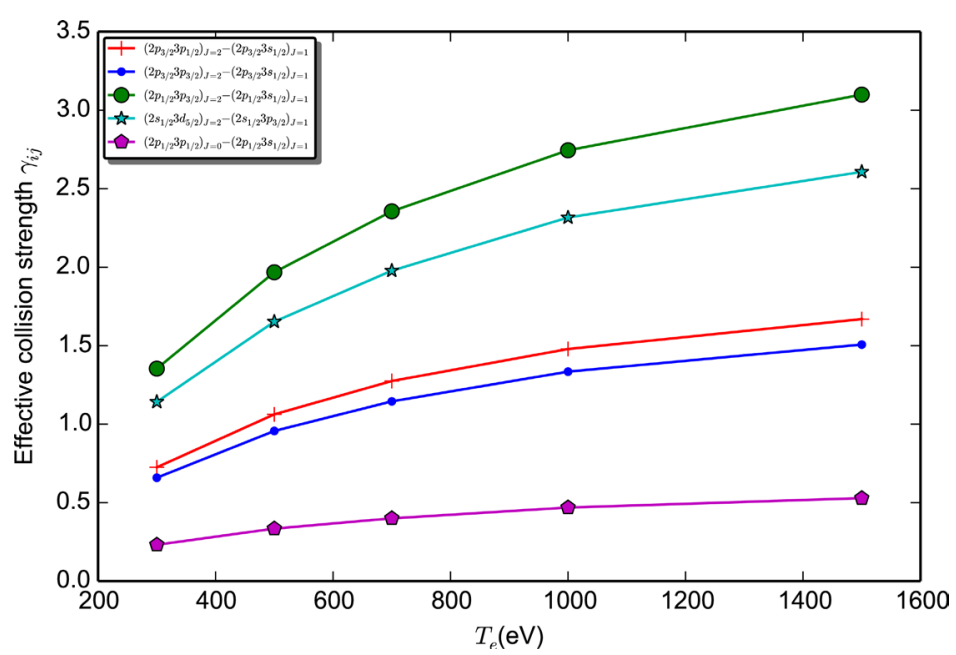

(a)

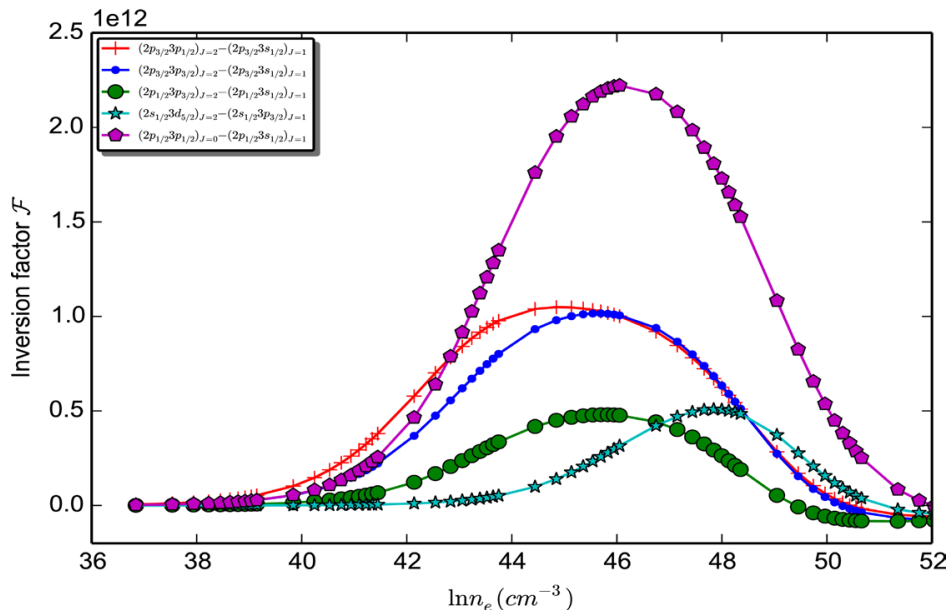

(b)

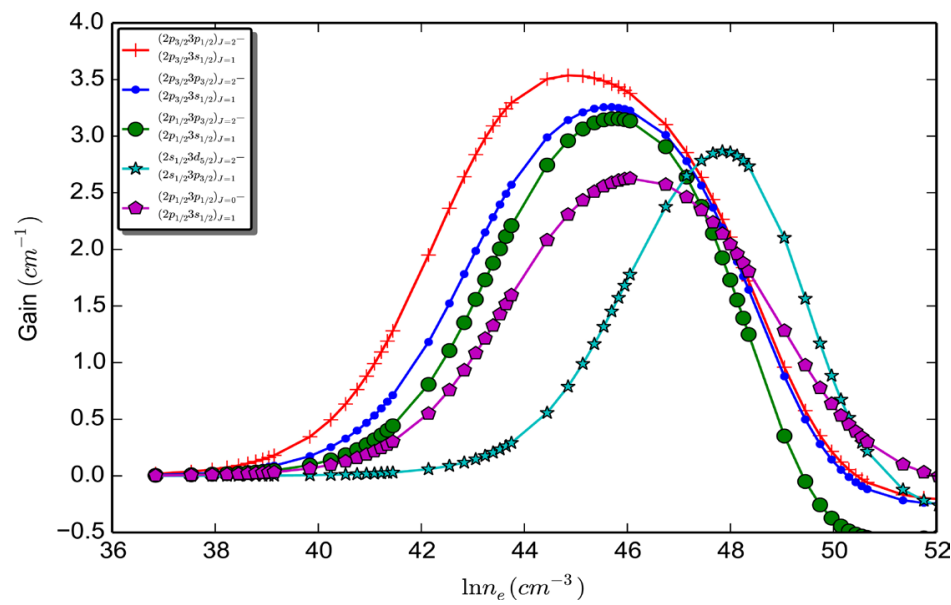

(c)

Figure 2. Effective collision strength, population inversion and gain coefficients for neon-like germanium ions $\mathrm{Ge}^{+22}$ at electron temperatures $T_{e}=700 \mathrm{eV}$. (a) Effective collision strengths of the Ne-like Ge at electron plasma temperatures $T_{e}=700 \mathrm{eV}$; (b) Population inversion factor $\mathcal{F}$ versus electron density $n_{e}$ at electron temperature $T_{e}=700 \mathrm{eV}$; (c) Gain coefficients at $T_{e}=700 \mathrm{eV}$ of the highest gain transitions in Neon like Germanium. 
Gain coefficients $g(v) \geq 1$ for both ions $\mathrm{Ga}^{+21}$ and $\mathrm{Ge}^{+22}$ are listed in Table 1 and Table 2 at five different electron plasma temperatures $\left(T_{e}=300,500,700,1000\right.$ and $\left.1500 \mathrm{eV}\right)$.

In neon like Gallium ions $\mathrm{Ga}^{+21}$, the following line transitions [17]-[19] ((10-2), (9-2) and (13-4)) as in Table 1 were detected with gains (4.3, 3.1 and $2.8 \mathrm{~cm}^{-1}$ ) respectively which agrees with our gains values except the transition (10-2) have higher gain value than our calculations. Also this line (8-2) was detected.

In neon like Germanium ions $G e^{+22}$, the following line transitions [19]-[23] ((9-2), (13-4), (34-30), (8-2) and (6-2)) as seen in Table 2 and the gain for (34-30) was detected to be $30 \mathrm{~cm}^{-1}$ which is greater than our calculations and other gains has a good agreement with our calculations.

Table 1. All wavelengths that appears at neon like Gallium $\mathrm{Ga}^{+21}$ at five electron plasma temperatures $T_{e}$. Data are ordered according to their wavelengths. The index column is the transition energy index and ranges from 0 to 456 , the transition column is the energy designation transition, $\lambda$ is the corresponding wavelength in $\mathrm{nm}$, the G-E column is the maximum gain $g(v)$ with the corresponding electron density $n_{e}$ written as $a(b) \equiv a \times 10^{b}$ and $T_{e}$ is the electron plasma temperature in $\mathrm{eV}$.

\begin{tabular}{|c|c|c|c|c|c|c|c|c|}
\hline \multirow{2}{*}{ Index } & \multirow{2}{*}{ Transition } & \multirow{2}{*}{$\lambda(\mathrm{nm})$} & \multirow{2}{*}{ G-E } & \multicolumn{5}{|c|}{$T_{e}(\mathrm{eV})$} \\
\hline & & & & 300 & 500 & 700 & 1000 & 1500 \\
\hline \multirow{2}{*}{$(14-2)$} & \multirow{2}{*}{$\left(2 p_{1 / 2} 3 p_{1 / 2}\right)_{J=0}^{e} \rightarrow\left(2 p_{3 / 2} 3 s_{1 / 2}\right)_{J=1}^{o}$} & \multirow{2}{*}{13.66} & $g(v)$ & & 1.1 & 1.54 & 1.83 & 1.92 \\
\hline & & & $n_{e}$ & & $1.0(20)$ & $1.0(20)$ & 8.0 (19) & 8.0 (19) \\
\hline \multirow{2}{*}{$(14-4)$} & \multirow{2}{*}{$\left(2 p_{1 / 2} 3 p_{1 / 2}\right)_{J=0}^{e} \rightarrow\left(2 p_{1 / 2} 3 s_{1 / 2}\right)_{J=1}^{o}$} & \multirow{2}{*}{19.408} & $g(v)$ & 1.16 & 3.22 & 4.5 & 5.34 & 5.6 \\
\hline & & & $n_{e}$ & $1.0(20)$ & $1.0(20)$ & 9.0 (19) & 8.0 (19) & $7.0(19)$ \\
\hline \multirow{2}{*}{$(10-2)$} & \multirow{2}{*}{$\left(2 p_{3 / 2} 3 p_{3 / 2}\right)_{J=0}^{e} \rightarrow\left(2 p_{3 / 2} 3 s_{1 / 2}\right)_{J=1}^{o}$} & \multirow{2}{*}{20.383} & $g(v)$ & & & 1.0 & 1.22 & 1.34 \\
\hline & & & $n_{e}$ & & & 4.0 (19) & $4.0(19)$ & $3.0(19)$ \\
\hline \multirow{2}{*}{$(34-30)$} & \multirow{2}{*}{$\left(2 s_{1 / 2} 3 d_{3 / 2}\right)_{J=2}^{e} \rightarrow\left(2 s_{1 / 2} 3 p_{1 / 2}\right)_{J=1}^{o}$} & \multirow{2}{*}{20.716} & $g(v)$ & & & 1.04 & 1.23 & 1.34 \\
\hline & & & $n_{e}$ & & & $2.0(20)$ & $2.0(20)$ & $3.0(20)$ \\
\hline \multirow{2}{*}{$(36-32)$} & \multirow{2}{*}{$\left(2 s_{1 / 2} 3 d_{5 / 2}\right)_{J=2}^{e} \rightarrow\left(2 s_{1 / 2} 3 p_{3 / 2}\right)_{J=1}^{o}$} & \multirow{2}{*}{20.951} & $g(v)$ & & 2.95 & 4.68 & 6.07 & 6.98 \\
\hline & & & $n_{e}$ & & $6.0(20)$ & $5.0(20)$ & $4.0(20)$ & $3.0(20)$ \\
\hline \multirow{2}{*}{$(20-8)$} & \multirow{2}{*}{$\left(2 p_{3 / 2} 3 d_{3 / 2}\right)_{J=2}^{o} \rightarrow\left(2 p_{3 / 2} 3 p_{3 / 2}\right)_{J=1}^{e}$} & \multirow{2}{*}{21.15} & $g(v)$ & & & 1.26 & 1.58 & 1.76 \\
\hline & & & $n_{e}$ & & & $2.0(20)$ & $2.0(20)$ & $2.0(20)$ \\
\hline \multirow{2}{*}{$(25-13)$} & \multirow{2}{*}{$\left(2 p_{1 / 2} 3 d_{5 / 2}\right)_{J=3}^{o} \rightarrow\left(2 p_{1 / 2} 3 p_{3 / 2}\right)_{J=2}^{e}$} & \multirow{2}{*}{21.647} & $g(v)$ & & & & & 1.27 \\
\hline & & & $n_{e}$ & & & & & $2.0(20)$ \\
\hline \multirow{2}{*}{$(9-2)$} & \multirow{2}{*}{$\left(2 p_{3 / 2} 3 p_{3 / 2}\right)_{J=2}^{e} \rightarrow\left(2 p_{3 / 2} 3 s_{1 / 2}\right)_{J=1}^{o}$} & \multirow{2}{*}{24.611} & $g(v)$ & 1.58 & 4.2 & 5.67 & 6.46 & 6.76 \\
\hline & & & $n_{e}$ & 8.0 (19) & 6.0 (19) & 5.0 (19) & 5.0 (19) & $5.0(19)$ \\
\hline$(13-4)$ & $(2 p-3 p-)^{e} \rightarrow(2 p-3 s)^{o}$ & 25.047 & $g(v)$ & 1.48 & 4.01 & 5.59 & 6.61 & 7.17 \\
\hline (7) & 12 & $20.07 \%$ & $n_{e}$ & 7.0 (19) & 6.0 (19) & $6.0(19)$ & $6.0(19)$ & $6.0(19)$ \\
\hline (12) & $(2 n-3 n)^{e}$ & 25505 & $g(v)$ & & 1.13 & 1.48 & 1.63 & 1.67 \\
\hline$(12-4)$ & $\left(2 p_{1 / 2} S p_{3 / 2}\right)_{J=1} \rightarrow\left(2 p_{1 / 2} S s_{1 / 2}\right)_{J=1}$ & 25.505 & $n_{e}$ & & 5.0 (19) & 5.0 (19) & $5.0(19)$ & $5.0(19)$ \\
\hline$(8-2)$ & $p^{e} \rightarrow(2 p-3 s)^{o}$ & 26.208 & $g(v)$ & 1.09 & 2.67 & 3.55 & 4.04 & 4.31 \\
\hline & $\left(2 P_{3 / 2} 2 P_{3 / 2}\right)_{J=1} \longrightarrow\left(2 P_{3 / 2} \mathrm{Ju}_{1 / 2}\right)_{J=1}$ & 20.200 & $n_{e}$ & 6.0 (19) & 5.0 (19) & 4.0 (19) & 4.0 (19) & 4.0 (19) \\
\hline$(11-4)$ & $(2 p-3 p)^{e} \quad(2 p-3 s)^{o}$ & 29735 & $g(v)$ & & 1.64 & 2.16 & 2.41 & 2.52 \\
\hline$(1+-7)$ & $\left(2 P_{1 / 2}-P_{1 / 2}\right)_{J=1} \rightarrow\left(2 P_{1 / 2} J_{1 / 2}\right)_{J=1}$ & 25.100 & $n_{e}$ & & $3.0(19)$ & 3.0 (19) & $3.0(19)$ & $3.0(19)$ \\
\hline$(6-2)$ & $\left(2 p^{3} 3 p\right)^{e} \rightarrow\left(2 p_{3} 3 s\right)^{o}$ & 29.919 & $g(v)$ & 1.74 & 4.57 & 6.18 & 7.07 & 7.46 \\
\hline & $\left(2 P_{3 / 2} U P_{1 / 2}\right)_{J=2}-1<P_{3 / 2}$ & & $n_{e}$ & $4.0(19)$ & $3.0(19)$ & 3.0 (19) & $3.0(19)$ & $3.0(19)$ \\
\hline$(290-266)$ & $(2 p-8 d)^{o} \rightarrow(2 p-8 p-)^{e}$ & 513.93 & $g(v)$ & & & & & 5.36 \\
\hline$(290-200)$ & $\left(2 P_{3 / 2}\right.$ & (513.50 & $n_{e}$ & & & & & $4.0(21)$ \\
\hline$(265-256)$ & $(2 p-8 p)^{e} \rightarrow(2 p-8 s)^{o}$ & 526.37 & $g(v)$ & & & & & 48.8 \\
\hline & $\left(2 P_{3 / 2} \mathrm{OP} P_{3 / 2}\right)_{J=3} \quad\left(2 P_{3}\right.$ & (5) & $n_{e}$ & & & & & $4.0(21)$ \\
\hline
\end{tabular}


Table 2. All wavelengths that appears at neon like Germanium $\mathrm{Ge}^{+22}$ at five electron plasma temperatures. The index column is the transition energy index and ranges from 0 to 456, the transition column is the energy designation transition, $\lambda$ is the corresponding wavelength in $\mathrm{nm}$, the G-E column is the maximum gain $g(v)$ with the corresponding electron density $n_{e}$ written as $a(b) \equiv a \times 10^{b}$ and $T_{e}$ is the electron plasma temperature in $\mathrm{eV}$.

\begin{tabular}{|c|c|c|c|c|c|c|c|c|}
\hline \multirow{2}{*}{ Index } & \multirow{2}{*}{ Transition } & \multirow{2}{*}{$\lambda(\mathrm{nm})$} & \multirow{2}{*}{ G-E } & \multicolumn{5}{|c|}{$T_{e}(\mathrm{eV})$} \\
\hline & & & & 300 & 500 & 700 & 1000 & 1500 \\
\hline \multirow{2}{*}{$(14-2)$} & \multirow{2}{*}{$\left(2 p_{1 / 2} 3 p_{1 / 2}\right)_{J=0}^{e} \rightarrow\left(2 p_{3 / 2} 3 s_{1 / 2}\right)_{J=1}^{o}$} & \multirow{2}{*}{12.741} & $g(v)$ & & & 1.05 & 1.3 & 1.42 \\
\hline & & & $n_{e}$ & & & $1.0(20)$ & $1.0(20)$ & $1.0(20)$ \\
\hline \multirow{2}{*}{$(14-4)$} & \multirow{2}{*}{$\left(2 p_{1 / 2} 3 p_{1 / 2}\right)_{J=0}^{e} \rightarrow\left(2 p_{1 / 2} 3 s_{1 / 2}\right)_{J=1}^{o}$} & \multirow{2}{*}{18.731} & $g(v)$ & & 2.29 & 3.41 & 4.24 & 4.6 \\
\hline & & & $n_{e}$ & & $1.0(20)$ & $1.0(20)$ & $1.0(20)$ & 9.0 (19) \\
\hline \multirow{2}{*}{$(10-2)$} & \multirow{2}{*}{$\left(2 p_{3 / 2} 3 p_{3 / 2}\right)_{J=0}^{e} \rightarrow\left(2 p_{3 / 2} 3 s_{1 / 2}\right)_{J=1}^{o}$} & \multirow{2}{*}{19.056} & $g(v)$ & & & & 1.12 & 1.27 \\
\hline & & & $n_{e}$ & & & & $5.0(19)$ & 4.0 (19) \\
\hline \multirow{2}{*}{$(34-30)$} & \multirow{2}{*}{$\left(2 s_{1 / 2} 3 d_{3 / 2}\right)_{J=2}^{e} \rightarrow\left(2 s_{1 / 2} 3 p_{1 / 2}\right)_{J=1}^{o}$} & \multirow{2}{*}{19.565} & $g(v)$ & & & & 1.07 & 1.27 \\
\hline & & & $n_{e}$ & & & & $3.0(20)$ & $3.0(20)$ \\
\hline \multirow{2}{*}{$(36-32)$} & \multirow{2}{*}{$\left(2 s_{1 / 2} 3 d_{5 / 2}\right)_{J=2}^{e} \rightarrow\left(2 s_{1 / 2} 3 p_{3 / 2}\right)_{J=1}^{o}$} & \multirow{2}{*}{19.979} & $g(v)$ & & 2.17 & 3.72 & 5.01 & 6.05 \\
\hline & & & $n_{e}$ & & $8.0(20)$ & $6.0(20)$ & $5.0(20)$ & $4.0(20)$ \\
\hline \multirow{2}{*}{$(20-8)$} & \multirow{2}{*}{$\left(2 p_{3 / 2} 3 d_{3 / 2}\right)_{J=2}^{o} \rightarrow\left(2 p_{3 / 2} 3 p_{3 / 2}\right)_{J=1}^{e}$} & \multirow{2}{*}{20.182} & $g(v)$ & & & 1.0 & 1.31 & 1.52 \\
\hline & & & $n_{e}$ & & & $2.0(20)$ & $2.0(20)$ & $2.0(20)$ \\
\hline \multirow{2}{*}{$(25-13)$} & \multirow{2}{*}{$\left(2 p_{1 / 2} 3 d_{5 / 2}\right)_{J=3}^{o} \rightarrow\left(2 p_{1 / 2} 3 p_{3 / 2}\right)_{J=2}^{e}$} & \multirow{2}{*}{20.663} & $g(v)$ & & & & & 1.12 \\
\hline & & & $n_{e}$ & & & & & $2.0(20)$ \\
\hline \multirow{2}{*}{$(9-2)$} & \multirow{2}{*}{$\left(2 p_{3 / 2} 3 p_{3 / 2}\right)_{J=2}^{e} \rightarrow\left(2 p_{3 / 2} 3 s_{1 / 2}\right)_{J=1}^{o}$} & \multirow{2}{*}{23.172} & $g(v)$ & & 2.97 & 4.23 & 5.01 & 5.4 \\
\hline & & & $n_{e}$ & & 8.0 (19) & 7.0 (19) & $6.0(19)$ & $6.0(19)$ \\
\hline \multirow{2}{*}{$(13-4)$} & \multirow{2}{*}{$\left(2 p_{1 / 2} 3 p_{3 / 2}\right)_{J=2}^{e} \rightarrow\left(2 p_{1 / 2} 3 s_{1 / 2}\right)_{J=1}^{o}$} & 23567 & $g(v)$ & & 2.78 & 4.1 & 5.02 & 5.66 \\
\hline & & 23.567 & $n_{e}$ & & 8.0 (19) & 7.0 (19) & 7.0 (19) & 8.0 (19) \\
\hline$(12-4)$ & $(2 n-3 n)^{e} \rightarrow(2 n-3 s)^{o}$ & 24015 & $g(v)$ & & & 1.08 & 1.24 & 1.31 \\
\hline & $\left(2 p_{1 / 2} S p_{3 / 2}\right)_{J=1} \rightarrow\left(2 p_{1 / 2} 3 S_{1 / 2}\right)_{J=1}$ & & $n_{e}$ & & & 6.0 (19) & $6.0(19)$ & $6.0(19)$ \\
\hline ) & $(2 n 3 n)^{e} \rightarrow(2 n-3 s)^{o}$ & 24712 & $g(v)$ & & 1.88 & 2.65 & 3.16 & 3.49 \\
\hline$(0-2)$ & $\left(2 P_{3 / 2} 3 P_{3 / 2}\right)_{J=1} \rightarrow(2 P$ & $24 . / 12$ & $n_{e}$ & & 6.0 (19) & 5.0 (19) & $5.0(19)$ & $5.0(19)$ \\
\hline$(11-4)$ & $(2 p-3 p)^{e} \rightarrow(2 p-3 s)^{\circ}$ & 28.41 & $g(v)$ & & 1.14 & 1.59 & 1.83 & 1.97 \\
\hline & $\left(2 P_{1 / 2} 3 P_{1 / 2}\right)_{J=1} \rightarrow\left(2 P_{1 / 2} 2 S_{1 / 2}\right)_{J=1}$ & & $n_{e}$ & & 4.0 (19) & 4.0 (19) & 4.0 (19) & 4.0 (19) \\
\hline$(6-2)$ & $(2 n-3 n)^{e} \rightarrow(2 n-3 s)^{o}$ & 28586 & $g(v)$ & 1.08 & 3.22 & 4.6 & 5.48 & 5.98 \\
\hline$(0-2)$ & $\left(-P_{3 / 2} \sigma P_{1 / 2}\right)_{J=2} \quad\left(-P_{3 / 2} \sigma_{1 / 2}\right)_{J=1}$ & 20.0000 & $n_{e}$ & 5.0 (19) & 4.0 (19) & 3.0 (19) & 3.0 (19) & 3.0 (19) \\
\hline
\end{tabular}

\section{Conclusion}

All possible transitions with gain coefficients $g(v) \geq 1$ are calculated for both neon-like gallium and germanium. Some of these lines were experimentally observed moreover shorter laser transitions are predicted from our calculations of the neon-like gallium and germanium ions. The collisional excitation model is sufficient to produce a population inversion and enough for explaining the emitted soft X-ray lasers.

\section{References}

[1] Hagelstein, P.L. (1981) Physics of Short-Wavelength-Laser Design.

[2] Matthews, D.L., Hagelstein, P., Rosen, M., Eckart, M., Ceglio, N., Hazi, A., Medecki, H., MacGowan, B., Trebes, J., Whitten, B., et al. (1985) Physical Review Letters, 54, 110. http://dx.doi.org/10.1103/PhysRevLett.54.110

[3] Matthews, D.L. and Rosen, M.D. (1988) Scientific American (USA), 259.

[4] Abdelaziz, W.S. and El Sherbini, T.M. (2010) Optics \& Laser Technology, 42, 699-702. 
http://dx.doi.org/10.1016/j.optlastec.2009.11.021

[5] Abdelaziz, W.S. (2011) Optics Communications, 284, 2859-2862. http://dx.doi.org/10.1016/j.optcom.2011.01.024

[6] El Sherbini, T.M. (1976) Zeitschrift für Physik A Atoms and Nuclei, 276, 325-327. http://dx.doi.org/10.1007/BF01548297

[7] Siegrist, M., Jia, F. and Balmer, J. (2016) Self-Photopumped X-Ray Lasers from Elements in the Ne-Like and Ni-Like Ionization State. $X$-Ray Lasers, Springer, 89-93.

[8] Calisti, A., Ferri, S., Mossé, C., Talin, B., Klisnick, A., Meng, L., Benredjem, D. and Guilbaud, O. (2014) Study of Particle Correlation Effects on Line Profiles of Ni-Like Collisional Xuv Laser Amplifier. X-Ray Lasers, Springer, 49-53.

[9] Meng, L., Klisnick, A., Kozlova, M., Bohacek, K., Krus, M., Prokupek, J., Urbanski, L., Marconi, M., Berrill, M., Rocca, J., et al. (2014) Temporal Coherence and Spectral Linewidth of Neon-Like Xuv Lasers Pumped in the QuasiSteady State Regime. X-Ray Lasers, Springer, 175-180.

[10] Urbanski, L., Marconi, M., Meng, L., Berrill, M., Guilbaud, O., Klisnick, A. and Rocca, J. (2014) Spectral Linewidth Measurement of a Ne-Like Ar Capillary Discharge Soft X-Ray Laser. X-Ray Lasers, Springer, 257-261.

[11] Yahia, M.E. and Azzouz, I.M. (2008) Optics \& Laser Technology, 1008-1017.

[12] Rosen, M., Hagelstein, P., Matthews, D., Campbell, E., Hazi, A., Whitten, B., MacGowan, B., Turner, R., Lee, R., Charatis, G., et al. (1985) Physical Review Letters, 54, 106. http://dx.doi.org/10.1103/PhysRevLett.54.106

[13] MacGowan, B., Da Silva, L., Fields, D., Keane, C., Koch, J., London, R., Matthews, D., Maxon, S., Mrowka, S., Osterheld, A., et al. (1992) Physics of Fluids B: Plasma Physics (1989-1993), 4, 2326-2337.

[14] Goldstein, W.H., Whitten, B.L., Hazi, A.U. and Chen, M.H. (1987) Physical Review A, 36, 3607. http://dx.doi.org/10.1103/PhysRevA.36.3607

[15] Gu, M.F. (2008) Canadian Journal of Physics, 86, 675-689. http://dx.doi.org/10.1139/P07-197

[16] Aggarwal, K.M. and Keenan, F.P. (2012) Atomic Data and Nuclear Data Tables, 98, 1003-1095. http://dx.doi.org/10.1016/j.adt.2011.05.002

[17] Fill, E.E., Li, Y. and Pretzler, G. (1995) IEEE Journal of Selected Topics in Quantum Electronics, 1, 958-961. http://dx.doi.org/10.1109/2944.473684

[18] Ditmire, T., Hutchinson, M., Key, M., Lewis, C., MacPhee, A., Mercer, I., Neely, D., Perry, M., Smith, R., Wark, J., et al. (1995) Physical Review A, 51, R4337. http://dx.doi.org/10.1103/PhysRevA.51.R4337

[19] McLean, E., Lee, T., Stamper, J., Manka, C. and Griem, H. (1992) JOSA B, 9, 350-359. http://dx.doi.org/10.1364/JOSAB.9.000350

[20] Fill, E.E., Li, Y., Preztler, G., Schlögl, D., Steingruber, J. and Nilsen, J. (1995) Physica Scripta, 52, 158. http://dx.doi.org/10.1088/0031-8949/52/2/003

[21] Warwick, P., Lewis, C., Kalachnikov, M., Nickles, P., Schnürer, M., Behjat, A., Demir, A., Tallents, G., Neely, D., Wolfrum, E., et al. (1998) JOSA B, 15, 1808-1814. http://dx.doi.org/10.1364/JOSAB.15.001808

[22] Enright, G., Kieffer, J., Herman, P., Pépin, H., Villeneuve, D., Dunn, J., Chaker, M. and Baldis, H. (1991) JOSA B, 8, 2047-2052. http://dx.doi.org/10.1364/JOSAB.8.002047

[23] Healy, S., Cairns, G., Lewis, C., Pert, G. and Plowes, J. (1995) IEEE Journal of Selected Topics in Quantum Electronics, 1, 949-957. http://dx.doi.org/10.1109/2944.473683 